\title{
Inclusion and Argument Properties for Certain Subclasses of Analytic Functions Defined by Using on Extended Multiplier Transformations
}

\author{
Oh Sang Kwon \\ Department of Mathematics, Kyungsung University, Busan, Korea \\ E-mail:oskwon@ks.ac.kr
}

Received March 28, 2011; revised April 27, 2011; accepted May 5, 2011

\begin{abstract}
Making use of a multiplier transformation, which is defined by means of the Hadamard product (or convolution), we introduce some new subclasses of analytic functions and investigate their inclusion relationships and argument properties.
\end{abstract}

Keywords: Subordination, Starlike Functions, Convex Functions, Closed-to-Convex Functions, Multiplier Transformation, Multivalent Functions, Argument Principle

\section{Introduction}

Let $A_{p}$ denote the class of functions $f$ normalized by

$$
f(z)=z^{p}+\sum_{k=1}^{\infty} a_{k+p} z^{k+p} \quad(p \in \mathbb{N}:=\{1,2,3, \cdots\})
$$

which are analytic and $p$-valent in the open unit disk

$$
U=\{z: z \in \mathbb{C} \text { and }|z|<1\}
$$

If $f$ and $g$ are analytic in $U$, we say that $f$ is subordinate to $g$, and write

$$
f \prec g \quad \text { or } \quad f(z) \prec g(z) \quad(z \in U)
$$

if there exists a Schwarz function $\omega(z)$, analytic in $U$ with $\omega(0)=0$ and $|\omega(z)|<1$ in $z \in U$, such that $f(z)=g(\omega(z))$ for $z \in U$.

We denote by $S_{p}^{*}(\eta)$ and $C_{p}(\eta)$ the subclasses of $A_{p}$ consisting of all analytic functions which are, respectively, $p$-valent starlike of order $\eta \quad(0 \leq \eta<p)$ in $U$ and $p$-valent convex of order $\eta \quad(0 \leq \eta<p)$ in $U$.

Let $M$ be the class of analytic functions $\varphi$ with $\varphi(0)=1$, which are convex and univalent in $U$ and satisfy the following inequality:

$$
\operatorname{Re}\{\varphi(z)\}>0 \quad(z \in U)
$$

Making use of the aforementioned principle of subordination between analytic functions, we define each of the following subclasses of $A_{p}$ :

$$
\begin{aligned}
& S_{p}^{*}(\eta ; \varphi) \\
& :=\operatorname{Re}\left\{f: f \in A_{p} \text { and } \frac{1}{p-\eta}\left(\frac{z f^{\prime}(z)}{f(z)}-\eta\right) \prec \varphi(z)\right\} \\
& (0 \leq \eta<p ; z \in U ; \varphi \in M)
\end{aligned}
$$

$$
\begin{array}{r}
K_{p}(\eta ; \varphi) \\
:=\operatorname{Re}\left\{f: f \in A_{p} \text { and } \frac{1}{p-\eta}\left(1+\frac{z f^{\prime \prime}(z)}{f^{\prime}(z)}-\eta\right) \prec \varphi(z)\right\} \\
\quad(0 \leq \eta<p ; z \in U ; \varphi \in M)
\end{array}
$$

For $m \in \mathbb{N}_{0}:=\{0,1,2, \cdots\}$, we define the multiplier transformation $J^{m}(p, \lambda, l)$ of functions $f \in A_{p}$ by

$$
\begin{gathered}
C_{p}(\eta, \beta ; \varphi, \psi):=\operatorname{Re}\left\{f: f \in A_{p} \text { and } \exists g \in S_{p}^{*}(\eta ; \varphi) \text { s.t. } \frac{1}{p-\beta}\left(\frac{z f^{\prime}(z)}{g(z)}-\beta\right) \prec \psi(z)\right\} \\
(0 \leq \eta, \beta<p ; z \in U ; \phi, \psi \in M)
\end{gathered}
$$




$$
\begin{array}{r}
J^{m}(p, \lambda, l) f(z)=z^{p}+\sum_{k=1}^{\infty}\left(\frac{l+\lambda k}{l}\right)^{m} a_{k+p} z^{k+p} \\
(l>0 ; \lambda \geq 0 ; z \in U)
\end{array}
$$

Put

$$
\begin{gathered}
\phi_{p, \lambda, l}^{m}(z)=z^{p}+\sum_{k=1}^{\infty}\left(\frac{l+\lambda k}{l}\right)^{m} z^{k+p} \\
(m \in \mathbb{N} ; l>0 ; \lambda \geq 0 ; z \in U)
\end{gathered}
$$

The operators $\phi_{p, \lambda, l}^{m}$ and $\phi_{p, 1, l}^{m}$, are the multiplier transformations introduced and studied earlier by Sarangi and Uralegaddi [16] and Uralegaddi and Somanatha ([1] and [2]), respectively. Correspending to the function $\phi_{p, \lambda, l}^{m}(z)$ defined by (1.6), we introduce a function $\phi_{p, \lambda, l}^{m, \mu}(z)$ given by the Hadamard product (or convolution):

$$
\phi_{p, \lambda, l}^{m}(z)^{*} \phi_{p, \lambda, l}^{m, \mu}(z)=\frac{z^{p}}{(1-z)^{\mu+p}} \quad(\mu>-p)
$$

Then, analogous to $J^{m}(p, \lambda, l)$, we have define a new multiplier transformation

$$
I_{\mu}^{m}(p, \lambda, l): A_{p} \rightarrow A_{p}
$$

as follows:

$$
I_{\mu}^{m}(p, \lambda, l) f(z)=\phi_{p, \lambda, l}^{m, \mu}(z) * f(z)
$$

We note that

$$
I_{-p}^{0}(p, 1,1) f(z)=f(z) \text { and } I_{2}^{1}(1,1,2) f(z)=z f^{\prime}(z)
$$

It is easily verifed from the above definition of the operator $I_{\mu}^{m}(p, \lambda, l)$, that

$$
\begin{aligned}
& z\left(I_{\mu}^{m}(p, \lambda, l) f(z)\right)^{\prime} \\
& =(\mu+p) I_{\mu+1}^{m}(p, \lambda, l) f(z)-\mu I_{\mu}^{m}(p, \lambda, l) f(z)
\end{aligned}
$$

and

$$
\begin{aligned}
& \lambda z\left(I_{\mu}^{m}(p, \lambda, l) f(z)\right)^{\prime} \\
& =l I_{\mu}^{m}(p, \lambda, l) f(z)-(\lambda p-l) I_{\mu}^{m+1}(p, \lambda, l) f(z)
\end{aligned}
$$

The definition (1.6) of the multiplier transformation $\phi_{p, \lambda, l}^{m}$ is motivated essentially by the Choi-SaigoSrivastava operator [3] for analytic functions, which includes a simpler integral operator studied earlier by Noor [7] and others (cf. [4-6]).

Next, by using the operator $I_{\mu}^{m}(p, \lambda, l)$ defined by (1.7), we introduce the following subclasses of analytic functions:

$$
\begin{aligned}
& S_{p, \lambda, l}^{m, \mu}(\eta ; \varphi) \\
& =\left\{f: f \in A_{p} \quad \text { and } \quad I_{\mu}^{m}(p, \lambda, l) f(z) \in S_{p}^{*}(\eta ; \varphi)\right\} \\
& \quad(\varphi \in M ; \lambda, l, \mu>0 ; m \in \mathbb{Z} ; 0 \leq \eta<1)
\end{aligned}
$$

$$
\begin{aligned}
& K_{p, \lambda, l}^{m, \mu}(\eta ; \varphi) \\
=\left\{f: f \in A_{p} \text { and } I_{\mu}^{m}(p, \lambda, l) f(z) \in K_{p}(\eta ; \varphi)\right\} & (\varphi \in M ; \lambda, l, \mu>0 ; m \in \mathbb{N} ; 0 \leq \eta<1)
\end{aligned}
$$

and

$$
\begin{aligned}
& C_{p, \lambda, l}^{m, \mu}(\eta, \beta ; \varphi, \psi) \\
=\left\{f: f \in A_{p} \text { and } I_{\mu}^{m}(p, \lambda, l) f(z) \in C(\eta, \beta ; \varphi, \psi)\right\} & (\varphi, \psi \in M ; \lambda, l, \mu>0 ; m \in \mathbb{N} ; 0 \leq \eta, \beta<1)
\end{aligned}
$$

We also note that

$$
f(z) \in K_{p, \lambda, l}^{m, \mu}(\eta ; \varphi) \Leftrightarrow z f^{\prime}(z) \in S_{p, \lambda, l}^{m, \mu}(\eta ; \varphi)
$$

In particular, we set

$$
S_{p, \lambda, l}^{m, \mu}\left(\eta ; \frac{1+A z}{1+B z}\right)=S_{p, \lambda, l}^{m, \mu}(\eta ; A, B) \quad(-1<B<A \leq 1)
$$

and

$$
K_{p, \lambda, l}^{m, \mu}\left(\eta ; \frac{1+A z}{1+B z}\right)=K_{p, \lambda, l}^{m, \mu}(\eta ; A, B) \quad(-1<B<A \leq 1)
$$

In the present paper, we investigate some inclusion relationships and argument properties associated with such multivalent functions in the class $A_{p}$ as those belonging to the subclasses $S_{p, \lambda, l}^{m, \mu}(\eta ; \varphi), K_{p, \lambda),}^{m, \mu}(\eta ; \varphi)$ and $C_{p, \lambda, l}^{m, \mu}(\eta, \beta ; \varphi, \psi)$ defined by (1.10), (1.11) and (1.12), respectively.

\section{Inclusion Properties}

Lemma 2.1: Let $\varphi$ be convex univalent in $U$ with $\varphi(0)=1$ and $\operatorname{Re}\{\beta \varphi(z)+v\}>0 \quad(\beta, v \in \mathbb{C})$. If $p$ is analytic in $U$ with $p(0)=1$, then

$$
p(z)+\frac{z p^{\prime}(z)}{\beta \varphi(z)+v} \prec \varphi(z) \quad(z \in U)
$$

implies that $p(z)<\varphi(z) \quad(z \in U)$.

Theorem 2.2: Let $\varphi \in M$ with

$$
\min _{z \in U}(\operatorname{Re}\{\varphi(z)\})>\max \left(\frac{\eta+\mu}{\eta-p}, \frac{\eta-p+\frac{l}{\lambda}}{\eta-p}\right)
$$


then $S_{p, \lambda, l}^{m, \mu+1}(\eta ; \varphi) \subset S_{p, \lambda, l}^{m, \mu}(\eta ; \varphi) \subset S_{p, \lambda, l}^{m+1, \mu}(\eta ; \varphi)$.

Proof. First of all, we show that

$S_{p, \lambda, l}^{m, \mu+1}(\eta ; \varphi) \subset S_{p, \lambda, l}^{m, \mu}(\eta ; \varphi)$. Let $f \in S_{p, \lambda, l}^{m, \mu+1}(\eta ; \varphi)$ and set

$$
p(z)=\frac{1}{p-\eta}\left(\frac{z\left(I_{\mu}^{m}(p, \lambda, l) f(z)\right)^{\prime}}{I_{\mu}^{m}(p, \lambda, l) f(z)}-\eta\right)
$$

where the function $p(z)$ is analytic in $U$ with $p(0)=1$.

Applying (2.1), we obtain

$$
(\mu+p) \frac{I_{\mu+1}^{m}(p, \lambda, l) f(z)}{I_{\mu}^{m}(p, \lambda, l) f(z)}=(p-\eta) p(z)+\eta+\mu
$$

By logarithmically differentiating both sides of (2.2) and multiplying the reseulting equation by $z$, we have

$$
\begin{aligned}
& \frac{1}{p-\eta}\left(\frac{z\left(I_{\mu}^{m}(p, \lambda, l) f(z)\right)^{\prime}}{I_{\mu}^{m}(p, \lambda, l) f(z)}-\eta\right) \\
& =p(z)+\frac{z p^{\prime}(z)}{(p-\eta) p(z)+\eta+\mu} \quad(z \in U)
\end{aligned}
$$

Since $\operatorname{Re}\{(p-\eta) \varphi(z)+\eta+\mu\}>0$, by applying Lemma 2.1 to (2.3), it follows that $p(z) \prec \varphi(z)$ in $U$, that is, that $f(z) \in S_{p, \lambda, l}^{m, \mu}(\eta ; \varphi)$.

To prove the second part of Theorem 2.1, let $f(z) \in S_{p, \lambda, l}^{m, \mu}(\eta ; \varphi)$ and put

$$
q(z)=\frac{1}{p-\eta}\left(\frac{z\left(I_{\mu+1}^{m}(p, \lambda, l) f(z)\right)^{\prime}}{I_{\mu+1}^{m}(p, \lambda, l) f(z)}-\eta\right)
$$

where the function $q(z)$ is analytic in $U$ with $q(0)=1$.

In precisely the same manner, we can find the result that $q(z) \prec \varphi(z)$ in $U$, that is, that $f(z) \in S_{p, \lambda, l}^{m+1, \mu}(\eta ; \varphi)$ under the hypothesis

$$
\operatorname{Re}\left\{(p-\eta) \varphi(z)+\eta-p+\frac{l}{\lambda}\right\}>0
$$

Theorem 2.3: Let $\varphi(z) \in M$ with

$$
\min _{z \in U}(\operatorname{Re}\{\varphi(z)\})>\max \left(\frac{\eta+\mu}{\eta-p}, \frac{\eta-p+\frac{l}{\lambda}}{\eta-p}\right)
$$

then $K_{p, \lambda, l}^{m, \mu+1}(\eta ; \varphi) \subset K_{p, \lambda, l}^{m, \mu}(\eta ; \varphi) \subset K_{p, \lambda, l}^{m+1, \mu}(\eta ; \varphi)$.

Proof. Applying (1.11) and Theorem 2.2, we observe that

$$
\begin{aligned}
& f(z) \in K_{p, \lambda, l}^{m, \mu+1}(\eta ; \varphi) \Leftrightarrow z f^{\prime}(z) \in S_{p, \lambda, l}^{m, \mu+1}(\eta ; \varphi) \\
& \Rightarrow z f^{\prime}(z) \in S_{p, \lambda, l}^{m, \mu}(\eta ; \varphi) \Leftrightarrow f(z) \in K_{p, \lambda, l}^{m, \mu}(\eta ; \varphi)
\end{aligned}
$$

and

$$
\begin{gathered}
f(z) \in K_{p, \lambda, l}^{m, \mu}(\eta ; \varphi) \Leftrightarrow z f^{\prime}(z) \in S_{p, \lambda, l}^{m, \mu}(\eta ; \varphi) \\
\Rightarrow z f^{\prime}(z) \in S_{p, \lambda, l}^{m+1, \mu}(\eta ; \varphi) \Leftrightarrow f(z) \in K_{p, \lambda, l}^{m+1, \mu}(\eta ; \varphi)
\end{gathered}
$$

which evidently prove Theorem 2.3 .

By setting

$$
\varphi(z)=\frac{1+A z}{1+B z} \quad(-1<B<A \leq 1 ; z \in U)
$$

in Theorems 2.2 and 2.3, we deduce the following corollary.

Corollary 2.4: Suppose that

$$
\frac{1-A}{1-B}>\max \left(\frac{\eta+\mu}{\eta-p}, \frac{\eta-p+\frac{l}{\lambda}}{\eta-p}\right)
$$

Then, for the function classes defined by (1.12) and (1.13),

$$
S_{p, \lambda, l}^{m, \mu+1}(\eta ; A, B) \subset S_{p, \lambda, l}^{m, \mu}(\eta ; A, B) \subset S_{p, \lambda, l}^{m+1, \mu}(\eta ; A, B)
$$

and

$$
K_{p, \lambda, l}^{m, \mu+1}(\eta ; A, B) \subset K_{p, \lambda, l}^{m, \mu}(\eta ; A, B) \subset K_{p, \lambda, l}^{m+1, \mu}(\eta ; A, B)
$$

Theorem 2.5: Let $\varphi, \psi \in M$ with

$$
\min _{z \in U}(\operatorname{Re}\{\varphi(z)\})>\max \left(\frac{\eta+\mu}{\eta-p}, \frac{\eta-p+\frac{l}{\lambda}}{\eta-p}\right)
$$

then

$$
\begin{aligned}
C_{p, \lambda, l}^{m, \mu+1}(\eta, \beta ; \varphi, \psi) & \subset C_{p, \lambda, l}^{m, \mu}(\eta, \beta ; \varphi, \psi) \\
& \subset C_{p, \lambda, l}^{m+1, \mu}(\eta, \beta ; \varphi, \psi)
\end{aligned}
$$

Proof. We begin by proving that $C_{p, \lambda, l}^{m, \mu+1}(\eta, \beta ; \varphi, \psi) \subset C_{p, \lambda, l}^{m, \mu}(\eta, \beta ; \varphi, \psi)$, which is the first inclusion relationship asserted by Theorem 2.5 .

Let $f(z) \in C_{p, \lambda, l}^{m, \mu+1}(\eta, \beta ; \varphi, \psi)$. Then there exists a function $k(z) \in S_{p}^{*}(\eta ; \varphi)$ such that

$$
\frac{1}{p-\beta}\left(\frac{z\left(I_{\mu+1}^{m}(p, \lambda, l) f(z)\right)^{\prime}}{k(z)}-\beta\right) \prec \psi(z) \quad(z \in U)
$$

Choose the function $g(z)$ such that

$$
I_{\mu+1}^{m}(p, \lambda, l) g(z)=k(z) \in S_{p}^{*}(\eta ; \varphi)
$$


Then $g(z) \in S_{p, \lambda, l}^{m, \mu+1}(\eta ; \varphi) \subset S_{p, \lambda, l}^{m, \mu}(\eta ; \varphi)$, and

$\frac{1}{p-\beta}\left(\frac{z\left(I_{\mu+1}^{m}(p, \lambda, l) f(z)\right)^{\prime}}{I_{\mu+1}^{m}(p, \lambda, l) g(z)}-\beta\right) \prec \psi(z) \quad(z \in U)$

Now let

$$
\left.\begin{array}{l}
\frac{1}{p-\beta}\left(\frac{z\left(I_{\mu+1}^{m}(p, \lambda, l) f(z)\right)^{\prime}}{I_{\mu+1}^{m}(p, \lambda, l) g(z)}-\beta\right)=\frac{1}{p-\beta} \cdot\left(\frac{z\left(z\left(I_{\mu}^{m}(p, \lambda, l) f(z)\right)^{\prime}+\mu I_{\mu}^{m}(p, \lambda, l) f(z)\right)^{\prime}}{z\left(I_{\mu}^{m}(p, \lambda, l) g(z)\right)^{\prime}+\mu I_{\mu}^{m}(p, \lambda, l) g(z)}-\beta\right) \\
=\frac{1}{p-\beta} \cdot\left(\frac{(\mu+1) z\left(I_{\mu}^{m}(p, \lambda, l) f(z)\right)^{\prime}+z^{2}\left(I_{\mu}^{m}(p, \lambda, l) f(z)\right)^{\prime \prime}}{z\left(I_{\mu}^{m}(p, \lambda, l) g(z)\right)^{\prime}+\mu I_{\mu}^{m}(p, \lambda, l) g(z)}-\beta\right) \\
=\frac{1}{p-\beta} \cdot\left(\frac{(\mu+1) \frac{z\left(I_{\mu}^{m}(p, \lambda, l) f(z)\right)^{\prime}}{I_{\mu}^{m}(p, \lambda, l) g(z)}+\frac{z^{2}\left(I_{\mu}^{m}(p, \lambda, l) f(z)\right)^{\prime \prime}}{I_{\mu}^{m}(p, \lambda, l) g(z)}}{\frac{z\left(I_{\mu}^{m}(p, \lambda, l) g(z)\right)^{\prime}}{I_{\mu}^{m}(p, \lambda, l) g(z)}+\mu}\right)
\end{array}\right)
$$

where the function $p(z)$ is analytic in $U$ with $p(0)=1$.

Using (1.9), we find that
Since $g(z) \in S_{p, \lambda, l}^{m, \mu}(\eta ; \varphi)$, then we set

$$
q(z)=\frac{1}{p-\eta}\left(\frac{z\left(I_{\mu}^{m}(p, \lambda, l) f(z)\right)^{\prime}}{I_{\mu}^{m}(p, \lambda, l) g(z)}-\eta\right)
$$

where $q(z) \prec \varphi(z)$ in $U$ with the assumption that $\varphi \in M$. By (2.5),

$$
\frac{z\left(I_{\mu}^{m}(p, \lambda, l) f(z)\right)^{\prime}}{I_{\mu}^{m}(p, \lambda, l) g(z)}=(p-\beta) p(z)+\beta
$$

Differentiating both side of (2.7) with respect to $z$ and multiplying by $z$, we obtain

$$
\begin{aligned}
& \frac{z\left(I_{\mu}^{m}(p, \lambda, l) f(z)\right)^{\prime \prime}}{\left(I_{\mu}^{m}(p, \lambda, l) f(z)\right)^{\prime}} \\
& =(p-\eta) q(z)+\eta-1+\frac{(p-\beta) z p^{\prime}(z)}{(p-\beta) p(z)+\beta}
\end{aligned}
$$

$$
\begin{aligned}
& \frac{1}{p-\beta}\left(\frac{z\left(I_{\mu+1}^{m}(p, \lambda, l) f(z)\right)^{\prime}}{I_{\mu+1}^{m}(p, \lambda, l) g(z)}-\beta\right) \\
& =\frac{1}{p-\beta}\left(\frac{(\mu+1)((p-\beta) p(z)+\beta)+(p-\beta) z p^{\prime}(z)+((p-\eta) q(z)+\eta-1)((p-\beta) p(z)+\beta)}{(p-\eta) q(z)+\eta+\mu}\right) \\
& =p(z)+\frac{z p^{\prime}(z)}{(p-\eta) q(z)+\eta+\mu}
\end{aligned}
$$

Hence

$$
\begin{aligned}
& \frac{z^{2}\left(I_{\mu}^{m}(p, \lambda, l) f(z)\right)^{\prime \prime}}{I_{\mu}^{m}(p, \lambda, l) g(z)} \\
= & (p-\beta) z p^{\prime}(z)+((p-\eta) q(z)+\eta-1) \\
& \cdot((p-\beta) p(z)+\beta)
\end{aligned}
$$

Computing the above equations, we can obtain 
Since $\operatorname{Re}\{(p-\eta) \varphi(z)+\eta+\mu\}>0$, applying Lemma

2.1 with $w(z)=\frac{1}{(p-\eta) \varphi(z)+\eta+\mu}$, we can show that $p(z) \prec \psi(z)$ in $U$, so that $f(z) \in C_{p, \lambda, l}^{m, \mu}(\eta, \beta ; \phi, \psi)$.

\section{Argument Properties}

Lemma 3.1: Let $\varphi$ be convex univalent in $U$ and $\omega$ be analytic in $U$ with $\operatorname{Re}\{\omega(z)\} \geq 0$. If $p(z)$ is analytic in $U$ and $p(0)=\varphi(0)$, then

$$
p(z)+\omega(z) z p^{\prime}(z) \prec \varphi(z) \quad(z \in U)
$$

implies that $p(z) \prec \varphi(z) \quad(z \in U)$.

Lemma 3.2: Let $p$ be analytic in $U$ with $p(0)=1$ and $p(z) \neq 0$ for all $z \in U$. If there exist two points $z_{1}, z_{2} \in U$ such that

$$
-\frac{\pi}{2} \alpha_{1}=\arg \left\{p\left(z_{1}\right)\right\}<\arg \{p(z)\}<\arg \left\{p\left(z_{2}\right)\right\}=\frac{\pi}{2} \alpha_{2}
$$

for some $\alpha_{1}$ and $\alpha_{2}\left(\alpha_{1}, \alpha_{2}>0\right)$ and for all $z$ $\left(|z|<\left|z_{1}\right|=\left|z_{2}\right|\right)$.

$\frac{z_{1} p^{\prime}\left(z_{1}\right)}{p\left(z_{1}\right)}=-i\left(\frac{\alpha_{1}+\alpha_{2}}{2}\right) m$ and $\frac{z_{2} p^{\prime}\left(z_{2}\right)}{p\left(z_{2}\right)}=i\left(\frac{\alpha_{1}+\alpha_{2}}{2}\right) m$

where $m \geq \frac{1-|b|}{1+|b|}$ and $b=i \tan \frac{\pi}{4}\left(\frac{\alpha_{2}-\alpha_{1}}{\alpha_{1}+\alpha_{2}}\right)$.

Theorem 3.3: Let $f \in A_{p} . \quad 0<\delta_{1}, \delta_{2} \leq 1 . \quad 0<\gamma<p$. If

$$
-\frac{\pi}{2} \delta_{1}<\arg \left(\frac{z\left(I_{\mu+1}^{m}(p, \lambda, l) f(z)\right)^{\prime}}{I_{\mu+1}^{m}(p, \lambda, l) g(z)}-\gamma\right)<\frac{\pi}{2} \delta_{2}
$$

for some $g \in S_{p, \lambda, l}^{m, \mu+1}(\eta, p ; A, B)$, then

$$
-\frac{\pi}{2} \alpha_{1}<\arg \left(\frac{z\left(I_{\mu}^{m}(p, \lambda, l) f(z)\right)^{\prime}}{I_{\mu}^{m}(p, \lambda, l) g(z)}-\gamma\right)<\frac{\pi}{2} \alpha_{2}
$$

where $\alpha_{1}, \alpha_{2}$ are the solutions for the following equations:

$$
\delta_{1}=\alpha_{1}+\frac{2}{\pi} \tan ^{-1}\left(\frac{\left(\alpha_{1}+\alpha_{2}\right)(1-|b|) \cos \left(\frac{\pi}{2} t_{1}\right)}{2\left(\frac{(p-\eta)(1+A)}{1+B}+\eta+\mu\right)(1+|b|)+\left(\alpha_{1}+\alpha_{2}\right)(1-|b|) \sin \left(\frac{\pi}{2} t_{1}\right)}\right)
$$

and

$$
\delta_{2}=\alpha_{2}+\frac{2}{\pi} \tan ^{-1}\left(\frac{\left(\alpha_{1}+\alpha_{2}\right)(1-|b|) \cos \left(\frac{\pi}{2} t_{1}\right)}{2\left(\frac{(p-\eta)(1+A)}{1+B}+\eta+\mu\right)(1+|b|)+\left(\alpha_{1}+\alpha_{2}\right)(1-|b|) \sin \left(\frac{\pi}{2} t_{1}\right)}\right)
$$

$b$ is given by (3.2), and

$$
t_{1}=t_{1}(\lambda)=\frac{2}{\pi} \cos ^{-1}\left(\frac{(p-\eta)(A-B)}{(p-\eta)(1-A B)+(\eta+\mu)\left(1-B^{2}\right)}\right)
$$

Proof. Let $p(z)=\frac{1}{p-\gamma}\left(\frac{z\left(I_{\mu}^{m}(p, \lambda, l) f(z)\right)^{\prime}}{I_{\mu}^{m}(p, \lambda, l) g(z)}-\gamma\right)$.

Then $p(z)$ is analytic in $U$ with $p(0)=1$. By using (1.9), we obtain

$$
\begin{aligned}
& ((p-\gamma) p(z)+\gamma)\left(I_{\mu}^{m}(p, \lambda, l) g(z)\right) \\
& =(\mu+p) I_{\mu+1}^{m}(p, \lambda, l) f(z)-\mu I_{\mu}^{m}(p, \lambda, l) f(z)
\end{aligned}
$$

Differentiating both sides of the above equation and multiplying the resulting equation by $z$, we find that

$$
\begin{aligned}
& (p-\gamma) p^{\prime}(z)\left(I_{\mu}^{m}(p, \lambda, l) g(z)\right) \\
& +((p-\gamma) p(z)+\gamma)\left(I_{\mu}^{m}(p, \lambda, l) g(z)\right)^{\prime} \\
& =(\mu+p)\left(I_{\mu+1}^{m}(p, \lambda, l) f(z)\right)^{\prime}-\mu\left(I_{\mu}^{m}(p, \lambda, l) f(z)\right)^{\prime}
\end{aligned}
$$

Since $g(z) \in S_{p, \lambda, l}^{m, \mu+1}(\eta, p ; A, B)$, by Corollary 2.4, it follows that $g(z) \in S_{p, \lambda, l}^{m, \mu}(\eta, p ; A, B)$.

Next we let $q(z)=\frac{1}{p-\eta}\left(\frac{z\left(I_{\mu}^{m}(p, \lambda, l) g(z)\right)^{\prime}}{I_{\mu}^{m}(p, \lambda, l) g(z)}-\eta\right)$. 
Then, using (1.9), we have

$$
(\mu+p) \frac{I_{\mu}^{m}(p, \lambda, l) g(z)}{I_{\mu}^{m}(p, \lambda, l) g(z)}=(p-\eta) q(z)+\eta+\mu
$$

From (3.4) and (3.5), we obtain

$$
\begin{aligned}
& \frac{1}{p-\gamma}\left(\frac{z\left(I_{\mu+1}^{m}(p, \lambda, l) f(z)\right)^{\prime}}{I_{\mu+1}^{m}(p, \lambda, l) g(z)}-\gamma\right) \\
& =p(z)+\frac{z p^{\prime}(z)}{(p-\eta) q(z)+\eta+\mu}
\end{aligned}
$$

Furthermore, by using a known result, we have

$$
\left|q(z)-\frac{1-A B}{1-B^{2}}\right|<\frac{A-B}{1-B^{2}}
$$

Thus, from (3.6), we obtain

$$
\begin{aligned}
& (p-\eta) q(z)+\eta+\mu=\rho \exp \left(\frac{i \pi}{2} \phi\right) \\
& \arg \left(p\left(z_{1}\right)+\frac{z_{1} p^{\prime}\left(z_{1}\right)}{(p-\eta) q\left(z_{1}\right)+\eta+\mu}\right) \\
& =-\frac{\pi}{2} \alpha_{1}+\arg \left(1-i\left(\frac{\alpha_{1}+\alpha_{2}}{2} m\left(\rho \exp \left(\frac{i \pi \phi}{2}\right)\right)^{-1}\right)\right) \leq-\frac{\pi}{2} \alpha_{1}-\tan ^{-1}\left(\frac{\left(\alpha_{1}+\alpha_{2}\right) m \cos \left(\frac{\pi}{2}(1-\phi)\right)}{2 \rho+\left(\alpha_{1}+\alpha_{2}\right) m \cos \left(\frac{\pi}{2}(1-\phi)\right)}\right) \\
& \leq-\frac{\pi}{2} \alpha_{1}-\tan ^{-1}\left(\frac{\left(\alpha_{1}+\alpha_{2}\right)(1-|b|) \cos \left(\frac{\pi}{2} t_{1}\right)}{2\left(\frac{(p-\eta)(1+A)}{1+B}+\eta+\mu\right)(1+|b|)+\left(\alpha_{1}+\alpha_{2}\right)(1-|b|) \cos \left(\frac{\pi}{2} t_{1}\right)}\right)=-\frac{\pi}{2} \delta_{1}
\end{aligned}
$$

we see that $\operatorname{Re}\{p(z)\}>0 \quad(z \in U)$, and hence $p(z) \neq 0 \quad(z \in U)$. By using Lemma 3.2, if there exist

and

$$
\arg \left(p\left(z_{2}\right)+\frac{z_{2} p^{\prime}\left(z_{2}\right)}{(p-\eta) q\left(z_{2}\right)+\eta+\mu}\right) \geq \frac{\pi}{2} \alpha_{2}+\tan ^{-1}\left(\frac{\left(\alpha_{1}+\alpha_{2}\right)(1-|b|) \cos \left(\frac{\pi}{2} t_{1}\right)}{2\left(\frac{(p-\eta)(1+A)}{1+B}+\eta+\mu\right)(1+|b|)+\left(\alpha_{1}+\alpha_{2}\right)(1-|b|) \cos \left(\frac{\pi}{2} t_{1}\right)}\right)=\frac{\pi}{2} \delta_{2}
$$

which would obviously contradict the assertion of Theorem 3.3. We thus complete the proof of Theorem 3.3.

If we let $\delta_{1}=\delta_{2}$ in Theorem 3.5, we easily obtain the following consequence.

Corollary 3.4: Let $f \in A_{p} . \quad 0<\delta \leq 1 . \quad 0<\gamma<p$. If

$$
\mid \arg \left(\frac{z\left(I_{\mu}^{m}(p, \lambda, l) f(z)\right)^{\prime}}{I_{\mu}^{m}(p, \lambda, l) g(z)}-\gamma\right)<\frac{\pi}{2} \delta
$$

for some $g \in S_{p, \lambda, l}^{m, \mu+1}(\eta, p ; A, B)$, then

$$
\begin{aligned}
& \left|\arg \left(\frac{z\left(I_{\mu}^{m}(p, \lambda, l) f(z)\right)^{\prime}}{I_{\mu}^{m}(p, \lambda, l) g(z)}-\gamma\right)\right| \\
& <\frac{\pi}{2} \alpha
\end{aligned}
$$

where $\alpha$ is the solutions for the following equation: 


$$
\delta=\alpha+\frac{2}{\pi} \tan ^{-1}\left(\frac{\alpha(1-|b|) \cos \left(\frac{\pi}{2} t_{1}\right)}{\left(\frac{(p-\eta)(1+A)}{1+B}+\eta+\mu\right)(1+|b|)+\alpha(1-|b|) \cos \left(\frac{\pi}{2} t_{1}\right)}\right)
$$

$b$ is given by (3.2), and

$$
-\frac{\pi}{2} \delta_{1}<\arg \left(\frac{z\left(I_{\mu}^{m}(p, \lambda, l) f(z)\right)^{\prime}}{I_{\mu}^{m}(p, \lambda, l) g(z)}-\gamma\right)<\frac{\pi}{2} \delta_{2}
$$

Theorem 3.5: Let $f \in A_{p} .0<\delta_{1}, \delta_{2} \leq 1 . \quad 0<\gamma<p$. If

$$
-\frac{\pi}{2} \delta_{1}<\arg \left(\frac{z\left(I_{\mu}^{m}(p, \lambda, l) f(z)\right)^{\prime}}{I_{\mu}^{m}(p, \lambda, l) g(z)}-\gamma\right)<\frac{\pi}{2} \delta_{2}
$$

for some $g \in S_{p, \lambda, l}^{m, \mu}(\eta, p ; A, B)$, then

$$
\begin{aligned}
& -\frac{\pi}{2} \alpha_{1}<\arg \left(\frac{z\left(I_{\mu}^{m+1}(p, \lambda, l) f(z)\right)^{\prime}}{I_{\mu}^{m+1}(p, \lambda, l) g(z)}-\gamma\right) \\
& <\frac{\pi}{2} \alpha_{2}
\end{aligned}
$$

where $\alpha_{1}, \alpha_{2}$ are the solutions for the following equations:

$$
\delta_{1}=\alpha_{1}+\frac{2}{\pi} \tan ^{-1}\left(\frac{\left(\alpha_{1}+\alpha_{2}\right)(1-|b|) \cos \left(\frac{\pi}{2} t_{1}\right)}{2\left(\frac{(p-\eta)(1+A)}{1+B}+\eta-p+\frac{l}{\lambda}\right)(1+|b|)+\left(\alpha_{1}+\alpha_{2}\right)(1-|b|) \cos \left(\frac{\pi}{2} t_{1}\right)}\right)
$$

and

$$
\delta_{2}=\alpha_{2}+\frac{2}{\pi} \tan ^{-1}\left(\frac{\left(\alpha_{1}+\alpha_{2}\right)(1-|b|) \cos \left(\frac{\pi}{2} t_{1}\right)}{2\left(\frac{(p-\eta)(1-A)}{1-B}+\eta-p+\frac{l}{\lambda}\right)(1+|b|)+\left(\alpha_{1}+\alpha_{2}\right)(1-|b|) \cos \left(\frac{\pi}{2} t_{1}\right)}\right)
$$

$b$ is given by (3.2), and

$$
\begin{aligned}
t_{1} & =t_{1}(\lambda) \\
& =\frac{2}{\pi} \cos ^{-1} \frac{(p-\eta)(A-B)}{(p-\eta)(1-A B)+\left(\eta-p+\frac{l}{\lambda}\right)\left(1-B^{2}\right)}
\end{aligned}
$$

$$
\left|\arg \left(\frac{z\left(I_{\mu}^{m}(p, \lambda, l) f(z)\right)^{\prime}}{I_{\mu}^{m}(p, \lambda, l) g(z)}-\gamma\right)\right|<\frac{\pi}{2} \delta
$$

for some $g \in S_{p, \lambda, l}^{m, \mu}(\eta, p ; A, B)$, then

If we let $\delta_{1}=\delta_{2}$ in Theorem 3.5, we easily obtain the following consequence.

Corollary 3.6: Let $f \in A_{p} . \quad 0<\delta \leq 1 . \quad 0<\gamma<p$. If

$$
\left|\arg \left(\frac{z\left(I_{\mu}^{m+1}(p, \lambda, l) f(z)\right)^{\prime}}{I_{\mu}^{m}(p, \lambda, l) g(z)}-\gamma\right)\right|<\frac{\pi}{2} \alpha
$$

where $\alpha$ is the solutions for the following equation:

$$
\delta=\alpha+\frac{2}{\pi} \tan ^{-1}\left(\frac{\alpha(1-|b|) \cos \left(\frac{\pi}{2} t_{1}\right)}{\left(\frac{(p-\eta)(1+A)}{1+B}+\eta-p+\frac{l}{\lambda}\right)(1+|b|)+\alpha(1-|b|) \cos \left(\frac{\pi}{2} t_{1}\right)}\right)
$$


$b$ is given by (1.17), and

$$
\begin{aligned}
t_{1} & =t_{1}(\lambda) \\
& =\frac{2}{\pi} \cos ^{-1}\left(\frac{(p-\eta)(A-B)}{(p-\eta)(1-A B)+\left(\eta-p+\frac{l}{\lambda}\right)\left(1-B^{2}\right)}\right)
\end{aligned}
$$

\section{Acknowledgements}

The research was supported by Kyungsung University Research Grants in 2011.

\section{References}

[1] B. A. Uralegaddi and C. Somanatha, "Certain Differential Operators for Meromorphic Functions," Houston Journal of Mathematics, Vol. 17, 1991, pp. 279-284.

[2] B. A. Uralegaddi and C. Somanatha, "New Critetia for Meromorphic Starlike Functions," Bulletin of the Australian Mathematical Society, Vol. 43, No. 1, 1991, pp. 137-140. doi:10.1017/S0004972700028859

[3] J. H. Choi, M. Saigo and H. M. Srivastava, "Some Inclusion Properties of a Certain Family of Integral Operators," Journal of Mathematical Analysis and Applications, Vol. 276, No. 1, 2002, pp. 432-445. doi:10.1016/S0022-247X(02)00500-0

[4] J.-L. Liu and K. I. Noor, "Some Properties of Noor Integral Operator," Journal of Natural Geometry, Vol. 21, 2002, pp. 81-90.

[5] J.-L. Liu, "The Noor Integral and Strongly Starlike Func- tions," Journal of Mathematical Analysis and Applications, Vol. 261, No. 2, 2001, pp. 441-447.

doi:10.1006/jmaa.2001.7489

[6] K. I. Noor and M. A. Noor, "On Integral Operators," Journal of Mathematical Analysis and Applications, Vol. 238, No. 2, 1999, pp. 341-352. doi:10.1006/jmaa.1999.6501

[7] K. I. Noor, "On New Classes of Integral Operators," Journal of Natural Geometry, Vol. 16, 1999, pp. 71-80.

[8] K. S. Padmanabhan and R. Parvatham, "On Analytic Functions and Differential Subordination," Bulletin Mathématique de la Société des Sciences, Mathématiques de Roumanie, Vol. 31, 1987, pp. 237-248.

[9] M. Nunokawa, S. Owa, H. Saitoh, N. E. Cho and N. Ta-kahashi, "Some Properties of Analytic Functions at Extremal Points for Arguments," preprint, 2003.

[10] P. Eenigenburg, S. S. Miller, P. T. Mocanu and M. O. Reade, "On a Briot-Bouquet Differential Subordination," General Inequalities, Vol. 3, 1983, pp. 339-348.

[11] R. J. Libera and M. S. Robertson, "Meromorphic Closeto-Convex Functions," Michigan Mathematical Journal, Vol. 8, No. 2, 1961, pp. 167-176. doi: $10.1307 / \mathrm{mmj} / 1028998568$

[12] S. K. Bajpai, "A Note on a Class of Meromorphic Univalent Functions," Revue Roumaine de Mathématiques Pures et Appliquées, Vol. 22, 1997, pp. 295-297.

[13] S. M. Sarangi and S. B. Uralegaddi, "Certain Differential Operators for Meromorphic Functions," Bulletin of the Calcutta Mathematical Society, Vol. 88, 1996, pp. 333-336

[14] S. S. Miller and P. T. Mocanu, "Differential Subordinations and Univalent Functions," Michigan Mathematical Journal, Vol. 28, No. 2, 1981, pp. 157-171. 